\title{
Genome-wide association study identifies new loci associated with noise-induced tinnitus in Chinese populations
}

\author{
Chengyong Xie', Yuguang Niu², Jie Ping ${ }^{3}$, Yahui Wang ${ }^{3}$, Chenning Yang ${ }^{3}$, Yuanfeng $\mathrm{Li}^{3 *}$ and Gangqiao Zhou ${ }^{1,3,4^{*}}$
}

\begin{abstract}
Background: Tinnitus is an auditory phantom sensation in the absence of an acoustic stimulus, which affects nearly $15 \%$ of the population. Excessive noise exposure is one of the main causes of tinnitus. To now, the knowledge of the genetic determinants of susceptibility to tinnitus remains limited.

Results: We performed a two-stage genome-wide association study (GWAS) and identified that two single nucleotide polymorphisms (SNPs), rs2846071 located in the intergenic region at 11q13.5 (odds ratio [OR] = 2.14, 95\% confidence interval $[\mathrm{Cl}]=1.96-3.40$, combined $P=4.89 \times 10^{-6}$ ) and rs4149577 located in the intron of TNFR SF1A gene at 12p13.31 $\left(\mathrm{OR}=2.05,95 \% \mathrm{Cl}=1.89-2.51\right.$, combined $\left.P=6.88 \times 10^{-6}\right)$, are significantly associated with the susceptibility to noise-induced tinnitus. Furthermore, the expression quantitative trait loci (eQTL) analyses revealed that rs 2846071 is significantly correlated with the expression of WNT11 gene, and rs4149577 with the expression of TNFRSF1A gene in multiple brain tissues (all $P<0.05$ ). The newly identified candidate gene WNT11 is involved in Wht pathway, and TNFRSFIA in the tumor necrosis factor pathway, respectively. Pathway enrichment analyses also showed that these two pathways are closely relevant to tinnitus.
\end{abstract}

Conclusions: Our findings highlight two novel loci at 11q13.5 and 12p13.31 conferring susceptibility to noiseinduced tinnitus. and suggest that the WNT11 and TNFRSFIA genes might be the candidate causal targets of $11 \mathrm{q} 13.5$ and 12p13.31 loci, respectively.

Keywords: Genome-wide association study, Tinnitus, Noise, WNT11, TNFRSF1A

\section{Background}

Tinnitus is an auditory phantom sensation in the absence of an acoustic stimulus, which affects nearly $15 \%$ of the population [1]. Tinnitus can result in an impossibility to relax and depression, which may seriously reduce the life quality of the affected individuals [2]. Therefore, understanding the mechanisms of tinnitus is of great significance. However, for decades, our knowledge of phantom sounds is limited, and the occurrence,

\footnotetext{
*Correspondence: liyf_snp@163.com; zhougq114@126.com

${ }^{3}$ State Key Laboratory of Proteomics, National Center for Protein Sciences, Beijing Institute of Radiation Medicine, Beijing 100850, China

Medical College of Guizhou University Guiyang City 550025 China

Full list of author information is available at the end of the article
}

development and clinical outcome of tinnitus remain largely unknown [3].

It has been reported that excessive noise exposure was one of the main causes of tinnitus [4-6]. Additionally, ototoxic drugs, hearing loss, stress, depression, sex, drinking, smoking and history of arthritis are also relevant to the development of tinnitus [4-6]. In addition to these external risk factors, genetic factors may also be involved in tinnitus susceptibility [7]. Recently, a longitudinal male twin cohort study $(n=1114$ at baseline and 583 at follow-up) confirmed that genetic factors do participate in the development of tinnitus [8]. Several candidate gene-based and genome-wide association studies (GWASs) have successfully identified a collection of

(c) The Author(s). 2021 Open Access This article is licensed under a Creative Commons Attribution 4.0 International License, which permits use, sharing, adaptation, distribution and reproduction in any medium or format, as long as you give appropriate credit to the original author(s) and the source, provide a link to the Creative Commons licence, and indicate if changes were made. The images or other third party material in this article are included in the article's Creative Commons licence, unless indicated otherwise in a credit line to the material. If material is not included in the article's Creative Commons licence and your intended use is not permitted by statutory regulation or exceeds the permitted use, you will need to obtain permission directly from the copyright holder. To view a copy of this licence, visit http://creativecommons.org/licenses/by/4.0/ The Creative Commons Public Domain Dedication waiver (http://creativecommons.org/publicdomain/zero/1.0/) applies to the data made available in this article, unless otherwise stated in a credit line to the data. 
candidate susceptibility genes for tinnitus, which can be roughly divided into the following five categories: (i) cardiovascular associated genes; (ii) neurotrophic factors associated genes; (iii) potassium recycling pathway genes; (iv) $\gamma$-aminobutyric acid type $\mathrm{B}\left(\mathrm{GABA}_{\mathrm{B}}\right)$ receptor subunit associated genes; and (v) serotonin receptor/transporter associated genes [9]. Especially, a recent GWAS of noise-induced tinnitus in the Belgian population showed that several metabolic pathways are significantly associated with this disease [10]. However, no GWAS for noise-induced tinnitus in the Chinese population has been performed.

To identify novel loci related to the risk of noiseinduced tinnitus in the Chinese population, we performed a GWAS; consisting of 65 noise-induced tinnitus patients (cases) and 233 subjects with normal hearing who have been exposed to a similar noise environment (controls), followed by a replication study in an independent sample set consisting of 34 cases and 379 controls. We found strong evidence for $11 \mathrm{q} 13.5$ and 12p13.31 as new loci contributing to susceptibility to noise-induced tinnitus. These findings expand our understanding of the genetic susceptibility to tinnitus.

\section{Results}

\section{Genome-wide association analyses}

To identify novel loci conferring susceptibility to noiseinduced tinnitus among Chinese populations, we performed a two-stage GWAS (Fig. 1). In the discovery stage, we used the Illumina Infinium Asian Screening Array-24 (v1.0) to genotype the 659,184 single nucleotide polymorphisms (SNPs) in 65 noise-induced tinnitus patients (cases) and 233 non-tinnitus individuals (controls) (Table 1 and Table S1). After quality controls, a total of 302,253 autosomal SNPs in these 298 individuals were retained, with an average genotyping call rate > 99.8\% (Table S2). No outlier was presented using the principal component analysis (PCA) (Fig. S1a). PCA also showed that the cases and controls are genetically wellmatched, and all these subjects are of Chinese ancestry (Fig. S1b). No significant principal components (PCs) were found using the Tracy-Widom statistic.

After imputation in strict accordance with the standard process, we achieved a total of 3,830,431 SNPs (Table S2). We then carried out genotype-phenotype association analyses by the logistic regression model, with adjustment for age and noise exposure time. A manhattan plot showed the associations between the genomewide SNPs and the risk of tinnitus (Fig. 2a). However, none of the SNPs reached the threshold for genomewide significance $\left(P<5.0 \times 10^{-8}\right)$. A quantile-quantile (Q-Q) plot was performed and showed a good match between the distributions of the observed $P$ values and those expected by chance (inflation factor $\lambda=1.003$; Fig.
$2 \mathrm{~b})$, suggesting minimal overall inflation of the genomewide statistical results in the discovery stage.

Several candidate gene-based association studies and a GWAS for tinnitus have identified several SNPs that were significantly associated with the risk of tinnitus [9]. In the present study, however, these SNPs did not show any significant association with noise-induced tinnitus (Table S3). These results are unlikely to be genotyping or imputation errors, since these SNPs were genotyped or imputed with high quality. The inconsistent associations may be due to the following reasons: (1) Population heterogeneity. The previously reported significantly associated SNPs in KCNE1 (index SNP rs915539) and KCTD12 (index SNP rs34544607) were monomorphic in the Chinese population (Table S3); (2) Limited sample sizes. The sample sizes in this study and most of the previous studies are less than 1000; (3) Different experimental designs. The experimental design in this study is to identify susceptibility genes in noise-induced tinnitus, while many previous studies aimed to identify susceptibility genes in other types of tinnitus, such as druginduced or age-induced tinnitus; and (4) Different sample collecting methods. The controls in this study are the non-tinnitus subjects exposing to the noise, while the controls in many previous studies are naïve controls.

\section{Two new susceptibility SNPs at $11 \mathrm{q} 13.5$ and 12p13.31 were identified}

We selected 22 index SNPs at 22 loci for replication in an independent population, which consists of 34 cases and 379 controls (Tables S4 and S5). Among these 22 index SNPs, two SNPs showed significant associations with the risk of tinnitus in the same direction as those observed in the discovery stage $(P=0.024$ for rs2846071 and $P=0.049$ for rs4149577, respectively; Table S6). In addition, we conducted meta-analyses for these two SNPs based on the results of the discovery and replication stages. Both SNPs showed more significant associations (odds ratio $[\mathrm{OR}]=2.14,95 \%$ confidence interval $[\mathrm{CI}]=1.96-3.40, \quad P=4.89 \times 10^{-6}$ for $\mathrm{rs} 2846071$; and $\mathrm{OR}=2.05, \quad 95 \% \quad \mathrm{CI}=1.89-2.51, \quad P=6.88 \times 10^{-6}$ for rs4149577; Table 2 and Fig. 3). No evidence for heterogeneity of OR values for rs2846071 and rs4149577 was observed across the populations from the discovery and replication stages $\left(P_{\text {heterogeneity }}=0.27\right.$ and 0.31 , respectively; Table 2).

We further investigated whether the age of subjects has a modification effect on the association between these two SNPs (rs2846071 and rs4149577) and tinnitus. We found no significant changes in the effects of rs2846071 and rs4149577 on the risk of noise-induced tinnitus when stratified by age $\left(P_{\text {heterogeneity }}=0.80\right.$ and 0.52 , respectively; Table S7). Since the participants in the discovery and replication stages of this study are all 


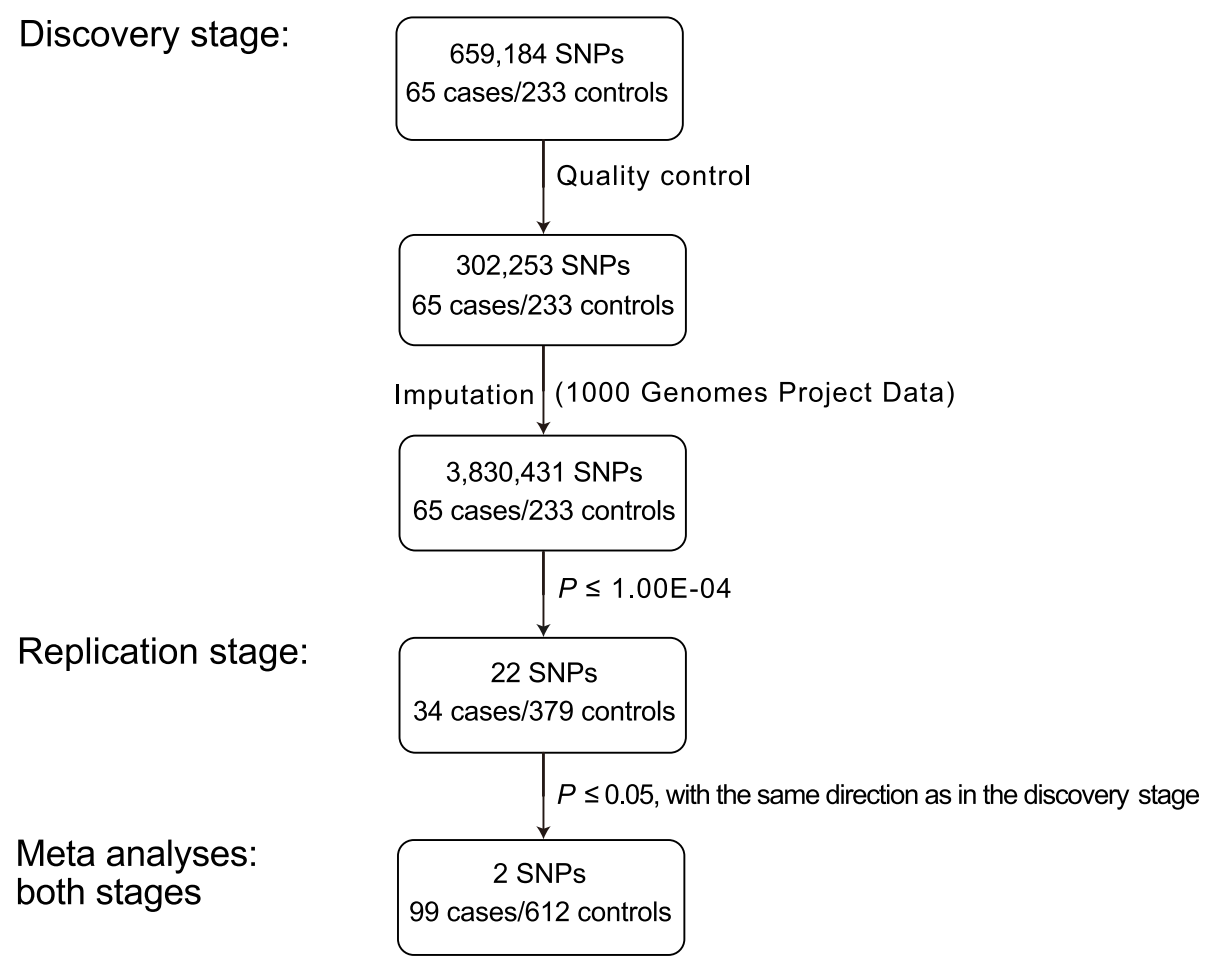

Fig. 1 An overview of the study workflow. Numbers refer to the sample sizes of the cases and controls, and the numbers of single nucleotide polymorphisms (SNPs) that were genotyped or imputed. The imputation was performed using the data from all populations from the 1000 Genomes Project (phase 3) and generated genotypes of a total of 3,830,431 SNPs. The 22 top significantly associated SNPs in the discovery stage were genotyped in the samples of the replication stage. Two SNPs, rs2846071 and rs4149577, were replicated in the replication stage. Lastly, meta-analyses combining two stages for rs2846071 and rs4149577 were performed

males, so the interference of sex-related factors could be ruled out.

\section{Chromosome 11q13.5 locus}

The index rs2846071 was located in the intergenic region at chromosome 11q13.5. Seven protein-coding genes (DGAT2, UVRAG, WNT11, THAP12, EMSY, LRRC32 and GUCY2EP) are located within the $500 \mathrm{~kb}$ region surrounding this SNP (Fig. 3a). We performed the expression quantitative trait loci (eQTL) analyses based on the datasets of brain tissues from the Genotype-Tissue Expression (GTEx, release v8) to identify the candidate causative genes at 11q13.5. We used eQTL data of the brain tissues, because several pieces of evidence have supported the relevance of brain tissues to tinnitus. For examples, several neuroscience studies have demonstrated that most tinnitus cases developed tinnitus as a consequence of changes that occur in central auditory pathways and other brain regions $[11,12]$. Moreover, the mice model studies have confirmed that tinnitus-related changes started in the cochlear nucleus and extended to the auditory cortex and other brain regions $[11,12]$. The eQTL analysis showed that the index rs2846071 is significantly associated with the expression levels of WNT11 in the brain anterior cingulate cortex, cerebellum and cortex $\left(P=0.047,7.7 \times 10^{-4}\right.$ and $1.5 \times$ $10^{-4}$, respectively; Fig. S2), but not with the expressions of other genes. We further performed colocalization analyses for GWAS and eQTL signals using the R package "Coloc" (3.2.1). However, the colocalization analyses showed that the posterior probability of hypothesis 4 (PP4) of rs2846071-WNT11 is less than 0.2 (PP4= 0.025), indicating that the tinnitus-associated SNP rs2846071 is not colocalized with eQTL signal for WNT11 in brain tissues (Figs. S3a-c). We also found that no nearby genes pass the PP4 threshold of $20 \%$ (data not shown). Indeed, WNT11 was identified as the top gene in the colocalization analyses. Taking this together with the significant eQTL results for WNT11 in the brain tissues, we suggested that WNT11 may be the candidate gene at this locus. Further studies are needed to confirm this hypothesis.

The causal SNP is not necessary the most statistically significant SNP [13]. Given this, we performed a functional annotation on the genetic variants tagged by index SNP rs2846071 to investigate the candidate causative variants at 11q13.5. Based on Haploreg (v4.1), eight SNPs at 11q13.5 are shown to be in strong or moderate 
Table 1 Summary of the case/control populations used in the discovery and replication stages

\begin{tabular}{|c|c|c|c|c|}
\hline \multirow[t]{2}{*}{ Stages } & \multicolumn{2}{|l|}{ Cases } & \multicolumn{2}{|l|}{ Controls } \\
\hline & Sample size & Mean age (s.d.) & Sample size & Mean age (s.d.) \\
\hline Discovery stage & 65 & 23.8 (1.6) & 233 & 23.4 (1.6) \\
\hline Replication stage & 34 & 26.4 (3.9) & 379 & $24.5(2.9)$ \\
\hline Overall & 99 & $24.7(2.9)$ & 612 & $24.1(2.5)$ \\
\hline
\end{tabular}

GWAS genome-wide association study, s.d standard deviation

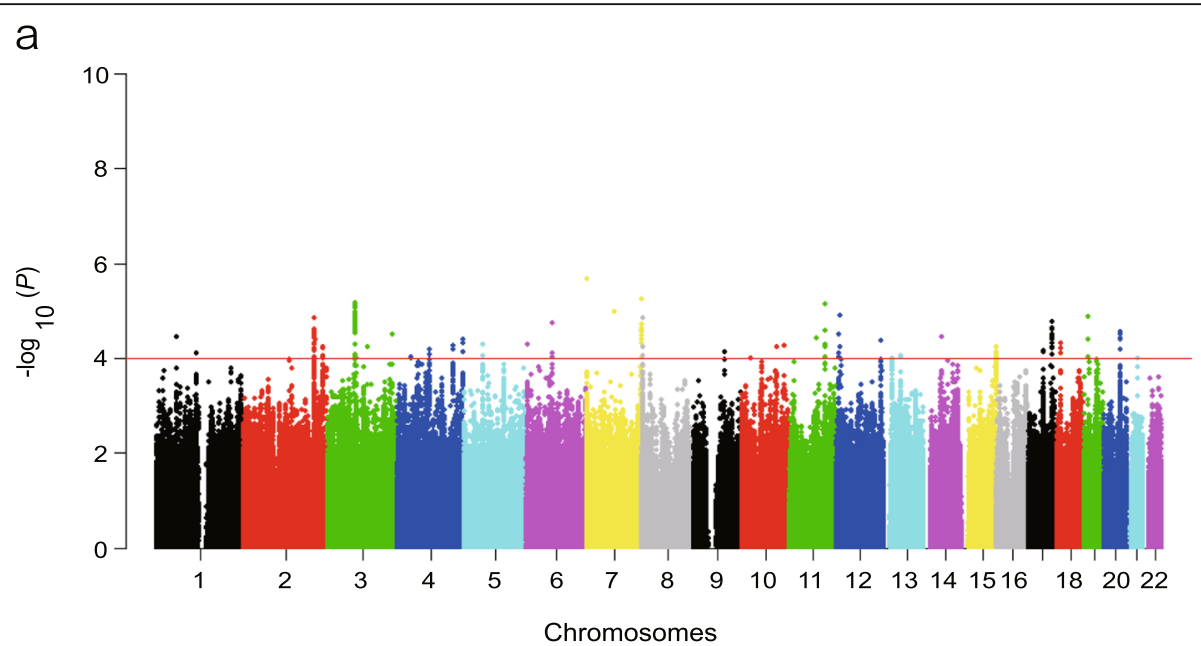

b

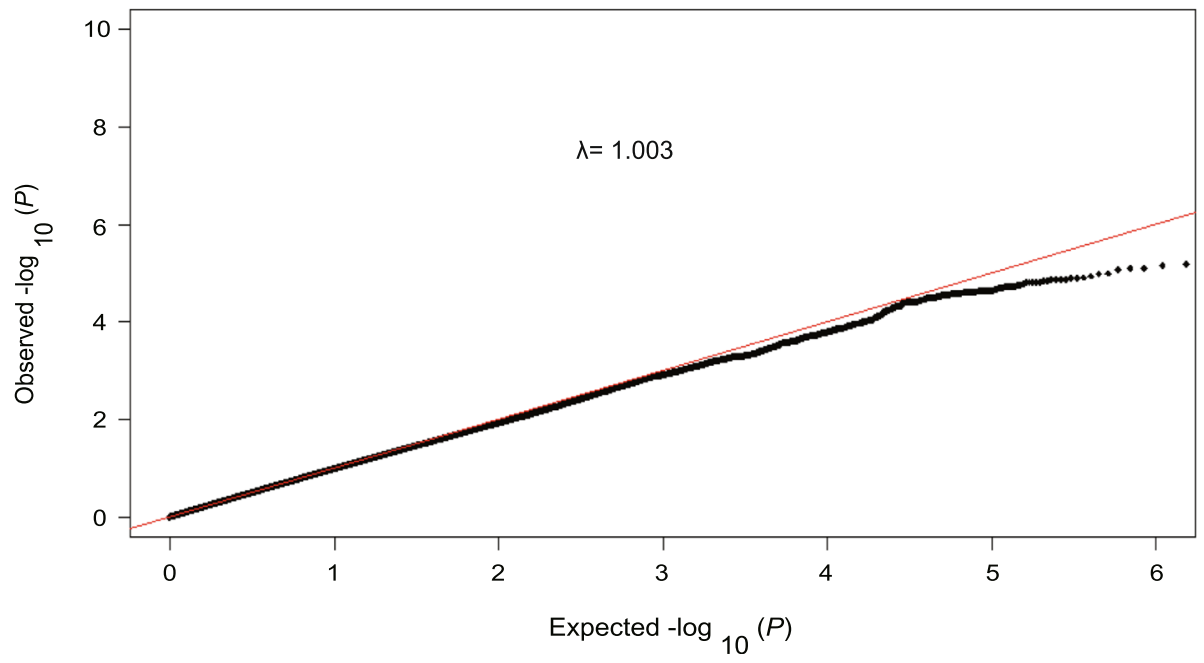

Fig. 2 Manhattan plot and Quantile-quantile plot of the genome-wide $P$ values from the association test on tinnitus. a The Manhattan plot of genome-wide $P$ values for the genotyped and imputed SNPs using logistic regression analyses in the cases/controls population in the discovery stage under the additive model. The $x$-axis represents the genomic position (based on NCBI Build 37), and the $y$-axis shows the -log10 (P). $\mathbf{b}$ The quantile-quantile plot. The red line represents the null hypothesis of no true association. The black line with gradient $\lambda$ (inflation coefficient) is fitted to the lower $90 \%$ of the distribution of the observed test statistics. The plot is based on the genotyped and imputed SNPs that passed the quality controls. The value of the inflation factor $\lambda$ is 1.003 under the additive model 
Table 2 Association results for the rs2846071 and rs4149577 in the case/control populations

\begin{tabular}{|c|c|c|c|c|c|c|c|c|}
\hline SNPs & Chr. (Cytoband) & Studies & Cases $^{a}$ & Controls $^{\mathrm{a}}$ & ORs $(95 \% \mathrm{Cls})$ & $P$ values & $I^{2}$ & $P_{\text {heterogeneity }}$ \\
\hline rs2846071 & $11 q 13.5$ & Discovery stage & $14 / 36 / 15$ & $16 / 107 / 110$ & $2.54(1.63-3.96)$ & $3.75 \times 10^{-5}$ & 19.07 & 0.27 \\
\hline \multirow[t]{2}{*}{$T / C^{b}$} & & Replication stage & $11 / 12 / 11$ & 50/161/157 & $1.75(1.08-2.84)$ & 0.024 & & \\
\hline & & Overall & $25 / 48 / 26$ & $66 / 277 / 267$ & $2.14(1.96-3.40)$ & $4.89 \times 10^{-6}$ & & \\
\hline rs4149577 & $12 \mathrm{p} 13.31$ & Discovery stage & 19/31/15 & 26/93/114 & $2.33(1.56-3.46)$ & $3.09 \times 10^{-5}$ & 1.28 & 0.31 \\
\hline \multirow[t]{2}{*}{$\mathrm{A} / \mathrm{G}^{\mathrm{b}}$} & & Replication stage & $8 / 16 / 10$ & 43/178/155 & $1.67(1.00-2.78)$ & 0.049 & & \\
\hline & & Overall & $27 / 47 / 25$ & 69/271/269 & $2.05(1.89-2.51)$ & $6.88 \times 10^{-6}$ & & \\
\hline
\end{tabular}

Chr. chromosome, $\mathrm{Cl}$ confidence interval, OR odds ratio, SNP single nucleotide polymorphism. ${ }^{\mathrm{a} C o u n t s}$ of TT/TC/CC genotypes for rs2846071 and AA/AG/GG genotypes for rs4149577 in the case/control populations, respectively. These two SNPs were genotyped using the Illumina Infinium Asian Screening Array-24 (v1.0) in the discovery stage. The number of genotyped samples varies due to genotyping failure. ${ }^{\mathrm{b}}$ Minor allele/major allele. ORs and $95 \%$ Cls were calculated under the additive model by logistic regression while adjusting for the age and noise exposure time

linkage disequilibrium (LD) with rs2846071 $\left(1 \geq r^{2} \geq 0.4\right)$, spanning $\sim 15 \mathrm{~kb}$ genomic regions (Table S8a). Using the PAINTOR software, we obtained the posterior probabilities for these 8 SNPs. Among these 8 SNPs, the rs2846071 has the highest posterior probability (0.48; Table S8a), suggesting that this SNP may be the causal SNP in this locus. Roadmap Epigenomics Consortium data revealed that rs2846071 is located in the enhancer region in multiple human brain tissues (Table S8a). Together, we thus speculated that rs2846071, or another in LD, may be the variant that has a causal effect on the risk of tinnitus by regulating WNT11 gene expression in brain tissues.

\section{Chromosome 12p13.31 locus}

The index SNPs rs4149577 was located at chromosome 12p13.31. More than 10 protein-coding genes were located in the $500 \mathrm{~kb}$ region surrounding rs4149577 (Fig. $3 \mathrm{~b})$. We performed eQTL analyses based on the 13 types of brain tissue in GTEx to identify the potentially causative gene(s) at 12p13.31. The eQTL analyses showed that the genotypes of rs4149577 are significantly associated with the expression levels of tumor necrosis factor receptor superfamily member $1 \mathrm{~A}$ (TNFRSF1A) in the brain caudate, cerebellar hemisphere, cerebellum, cortex, frontal cortex and putamen tissues (all $P<0.05$; Fig. S4). Colocalization analysis further showed that the tinnitusassociated SNP rs4149577 is colocalized with eQTL signals for TNFRSF1A in brain tissues $(\mathrm{PP} 0=0.040, \mathrm{PP} 1=$ 0.002, PP2 $=0.613$, PP3 $=0.057$, PP $4=0.288$; Figs. S3d-f) [14]. Together, these pieces of evidence suggested a potential role for TNFRSF1A in the development of tinnitus.

To identify the potential causal variants at the 12p13.31 locus, we performed a functional annotation on the 8 SNPs tagged by index SNP rs4149577 $\left(r^{2}>0.4\right)$, which span $\sim 14 \mathrm{~kb}$ region (Table S8b). By using the PAINTOR software, we got the posterior probability of these 8 SNPs at 12p13.31. Among them, rs1800692 and rs4149570 had the highest posterior probability (1.00;
Table S8b). We performed eQTL analyses for these two SNPs and showed that the genotypes of these two SNPs are significantly associated with TNFRSF1A in multiple brain tissues (all $P<0.05$ ). The most significant eQTL results for these two SNPs were in the brain caudate tissues $\left(P=1.9 \times 10^{-5}\right.$ and $4.8 \times 10^{-5}$, respectively). Further, these two SNPs were predicted to be located in enhancer and promoter signals in human brain tissues based on Roadmap Epigenomics Consortium data (Table S8b). Additionally, chromatin state segmentation by hidden markov model (HMM) from ENCODE/Broad database showed that these two SNPs are located in enhancer regions in various types of cells (Fig. S5). Together, these results suggested that these two SNPs may be the candidate causative variants in this region.

\section{Pathway enrichment analyses}

To investigate the pathways or biological processes potentially involved in noise-induced tinnitus, we employed the i-GSEA4GWAS, which is a tool using the summary statistics of all SNPs from the GWAS, without restricting the analyses to a significance threshold [15]. In total, five pathways showed significant associations with noiseinduced tinnitus, including the arachidonic acid metabolism, inositol phosphate metabolism, Notch signaling, Wnt signaling and tumor necrosis factor (TNF) pathways (all $P<0.05$; Table S9). Among these pathways, the arachidonic acid metabolism and inositol phosphate metabolism showed the strongest association $(P<0.001$; Table S9). This result was consistent with previous GWAS findings that the metabolic pathways were significantly associated with tinnitus [10]. It has been reported that the altered arachidonic acid (a substrate of cyclooxygenase) metabolism may be the physiological basis of salicylate-induced tinnitus [16]. The inositol phosphate has been shown to induce $\mathrm{Ca}^{2+}$ elevation in cochlear sensory epithelial cells [17]. Additionally, several other pathways may be involved in tinnitus. For example, Wnt signaling and Notch signaling could regulate each other [18, 19], and are required for 
a

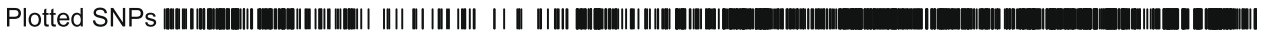

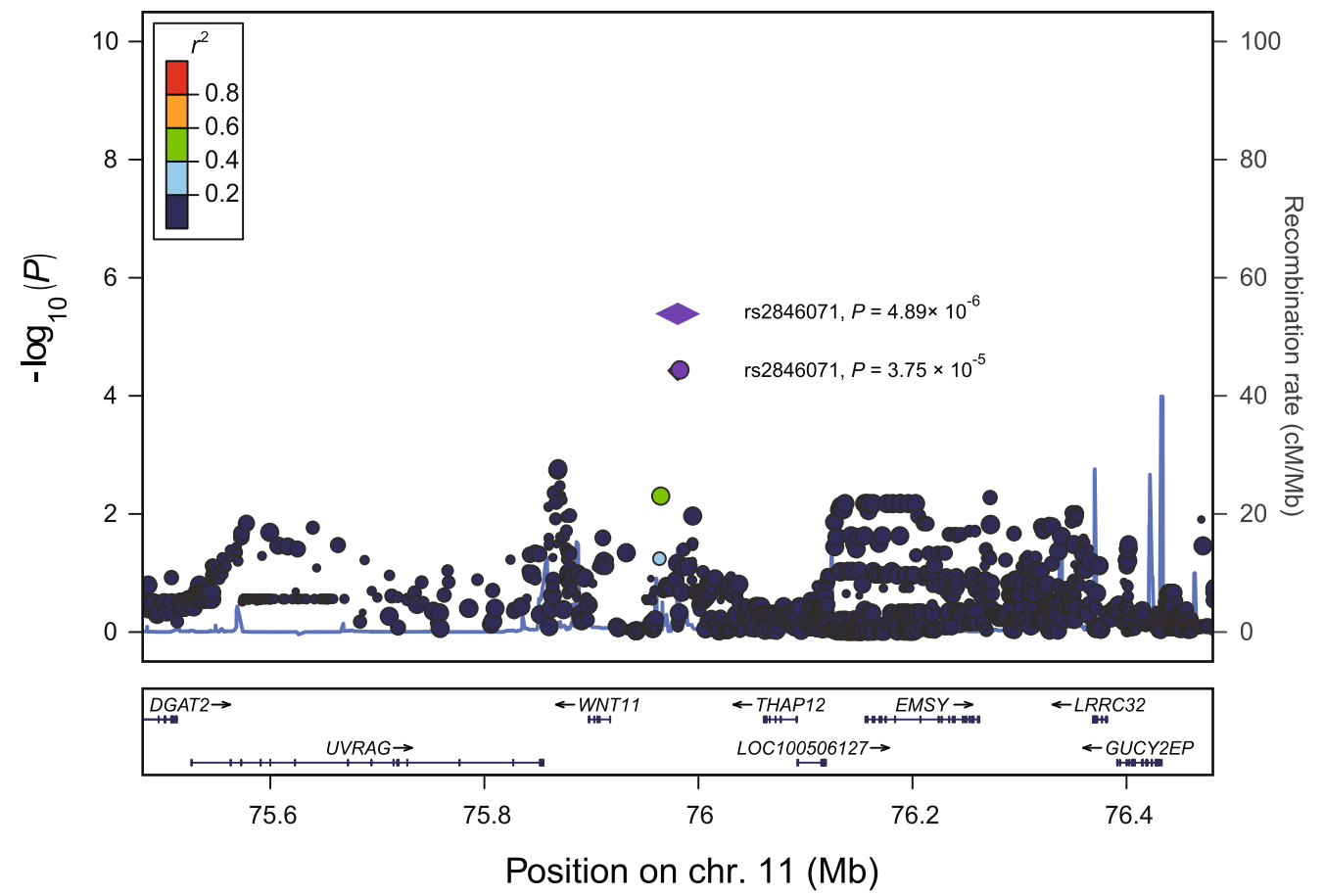

b

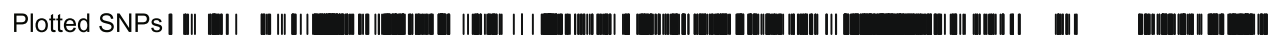

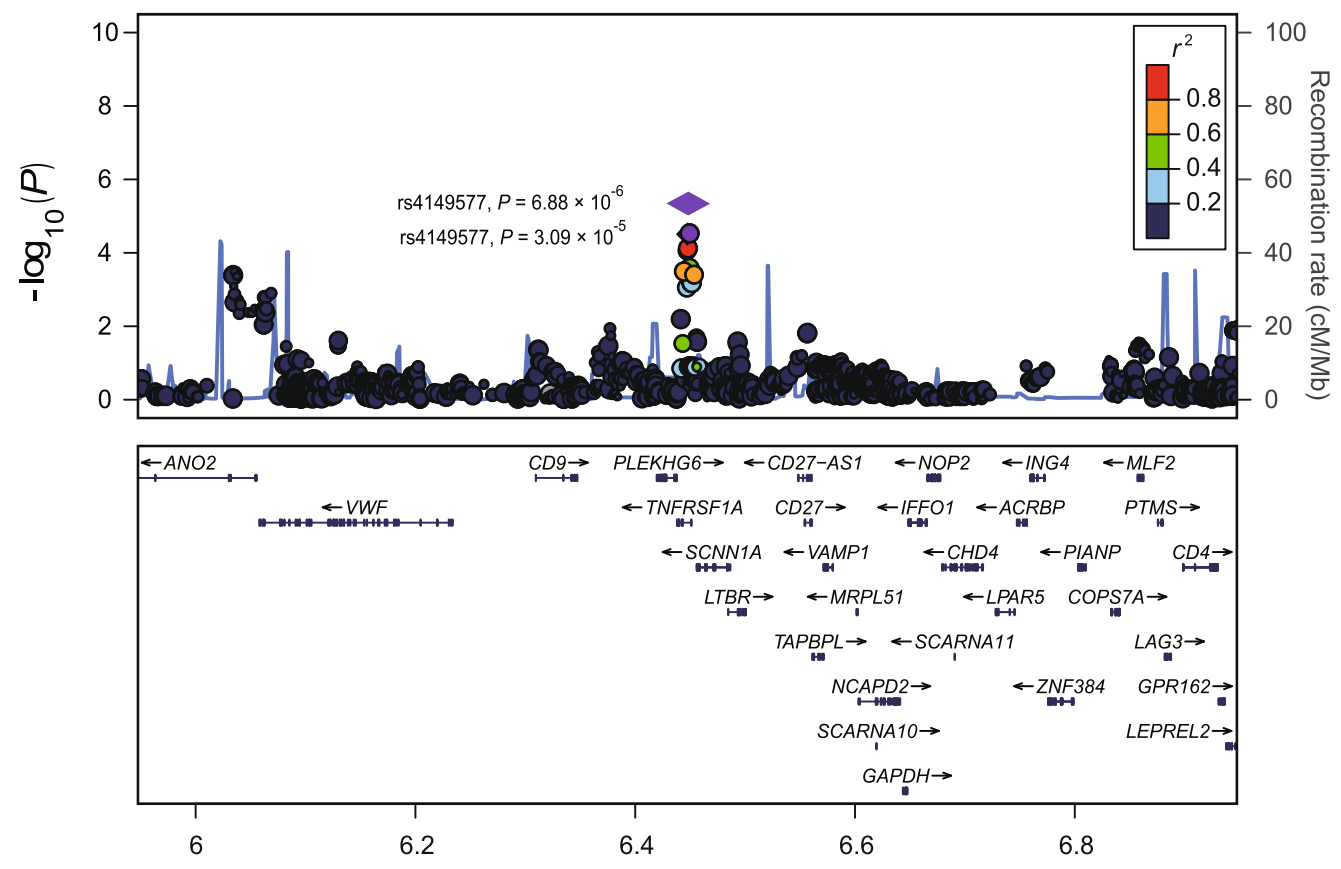

Position on chr. $12(\mathrm{Mb})$

Fig. 3 (See legend on next page.) 
(See figure on previous page.)

Fig. 3 Regional plots for the associations in regions surrounding the rs2846071 or rs4149577 in the discovery stage. Genomic positions are based on NCBI Build 37. In the meta-analysis, the $P$ value of the SNP is shown as purple diamonds, with their initial $P$ value in the discovery stage shown as purple dots. The linkage disequilibrium (LD) values $\left(r^{2}\right)$ to rs2846071 or rs4149577 for the other SNPs are indicated by marked color. Red signifies $r^{2}>0.8$, orange $0.6<r^{2} \leq 0.8$, green $0.4<r^{2} \leq 0.6$, light blue $0.2<r^{2} \leq 0.4$ and blue $r^{2} \leq 0.2$. Estimated recombination rates, which are derived from the East Asian populations of the 1000 Genomes Project (phase 3), are plotted in blue. Genes within the 500 kb region surrounding the index SNPs rs2846071 (a) or rs4149577 (b) are annotated, with the positions of transcripts shown by arrows. The East Asian populations from the 1000 Genomes Project consist of 504 subjects from the CHB (Han Chinese in Beijing, China), CHS (Southern Han Chinese), CDX (Chinese Dai in Xishuangbanna, China), JPT (Japanese in Tokyo, Japan) and KHV (Kinh in Ho Chi Minh City, Vietnam)

supporting cell proliferation and hair cell differentiation in the cochlea [20]. TNF pathway could induce apoptosis of auditory hair cells in vitro in hair cell nuclei [21]. Intriguingly, the newly identified significantly associated genes WNT11 and TNFRSF1A were just in the Wnt and TNF pathways, respectively, therefore highlight the critical roles of these two pathways in the development of tinnitus.

\section{Discussion}

In the present study, we performed a GWAS of noiseinduced tinnitus in the Chinese population. To our best knowledge, this is the first GWAS for the risk of tinnitus among Chinese population. We successfully identified two novel loci at 11q13.5 (index rs2846071) and 12p13.31 (index rs4149577) loci, which were significantly associated with the susceptibility to noise-induced tinnitus.

We compared the allele frequencies of these two SNPs with those in the main populations from the $1000 \mathrm{Ge}$ nomes Project (phase 3). On the one hand, we found that the allele frequency of rs2846071 [T] (0.356) is similar to that of East Asian descent (0.354, $P=0.94$ ) in the 1000 Genomes Project, but significantly lower than that of Europeans, Africans and Americans descent $(P=$ $1.56 \times 10^{-53}, 2.39 \times 10^{-110}$ and $1.97 \times 10^{-15}$, respectively; Table S10). On the other hand, we found that the SNP rs4149577 [A] allele frequency (0.360) is similar to that of the East Asian descent $(0.360, P=0.77)$, but significantly lower than that of the Europeans, Africans and Americans descent $\left(P=2.15 \times 10^{-16}, 2.25 \times 10^{-204}\right.$ and $1.64 \times 10^{-8}$, respectively; Table S10). Further studies are needed to investigate whether the difference in allele frequencies of these SNPs among different ethnic groups affects the susceptibility to tinnitus.

The two identified SNPs in this study are all located in non-coding regions, which may affect the disease risk by regulating the transcription levels of related genes [22] The eQTL analysis is helpful to reveal the relationship between the genetic variation and expression of the nearby genes in specific tissue types [23]. Here, our eQTL analyses showed that the genotypes of rs2846071 or rs4149577 are correlated with the expression levels of
WNT11 or TNFRSF1A, respectively, in multiple brain tissues, suggesting that these two genes may be the candidate genes of tinnitus. However, the analyses of eQTL were complicated due to tissue heterogeneity. In addition, most of the samples in the GTEx database are of European ancestry, while the samples in this GWAS were all of Chinese ancestry. The differences in LD values and allele frequencies between the Chinese population and the European population will potentially influence the eQTL and colocalization signals (Figs. S6 and S7). Thus, it is necessary to be cautious when interpreting the results of the eQTL and colocalization analyses. Further analysis was needed in a larger sample size study to confirm the genetic associations of WNT11 and TNFRSF1A with tinnitus.

The WNT11 gene encodes a secretory signal protein, which is involved in the Wnt pathway [24]. WNT11 has been reported to involve the formation of cilia [25], and the abnormality of cilia could influence tinnitus occurrence [26]. Besides, the Wnt pathway was considered to be the key to many basic development processes, including the hearing items [27]. For example, knockout of $\beta$-catenin in mice has been shown to inhibit the differentiation of hair cells as well as columnar cells from sensory progenitor cells [28]. Besides, Wnt activation could protect against hair cell damage in the mouse cochlea [29]. Mutations in several genes of the Wnt/planar cell polarity (PCP) pathway (such as Wnt11 and Gpc4) could result in the misorientation of hair cells in mice [30]. TNFRSF1A encodes a member of TNF receptor superfamily of proteins and was involved in TNF pathway [31]. TNFRSF1A has been shown to involve in the production of ototoxic reactive oxygen species and has been demonstrated to be specifically upregulated in gentamicin-mediated ototoxicity [32], suggesting that high expression of TNFRSF1A may have damaging effects on hearing and hair cells [32]. Besides, TNFRSF1A may cause infiltration of inflammatory cells, which is known to be the main cause of hearing problems [33]. As for the TNF pathway, genetic knockout of tumor necrosis factor-alpha (TNF- $\alpha$ ) or pharmacologically blocking TNF- $\alpha$ expression ameliorated the behavioral phenotype associated with noise-induced tinnitus in 
mice [34]. Together, these pieces of evidence suggested potential roles for WNT11 and TNFRSF1A in the development of tinnitus.

We also performed meta-analyses for all of the 22 candidate SNPs in the discovery and replication stages. We found that in addition to the reported two SNPs rs2846071 and rs4149577, another SNP rs10771523 also shows a more significant association in the meta-analysis $\left(P=1.70 \times 10^{-6}\right)$ than that in the discovery stage. However, the association between rs10771523 and tinnitus in the replication stage was not significant $(P=0.26)$. Further genetic association studies are needed to replicate whether rs10771523 is significantly associated with tinnitus.

In the pathway analyses, we noted that the top two enriched pathways are metabolism pathways: arachidonic acid metabolism and inositol phosphate metabolism. These findings are consistent with a previous GWAS, which also suggested that several metabolic pathways (such as serotonin reception mediated signaling) are significantly associated with tinnitus $[10,35]$. Indeed, tinnitus is considered to be a result of metabolic, neurologic and psychogenic disorders [36]. Thus, our results further highlighted the important roles of metabolism pathways in the development of tinnitus.

Up to now, two GWASs of tinnitus in European populations have been reported [10,37]. The first GWAS of tinnitus consists of 167 tinnitus subjects and 749 nontinnitus subjects [10], and none of the SNPs reach the threshold for genome-wide significance $\left(P<5.0 \times 10^{-8}\right)$. However, several metabolic pathways showed significant associations. The other GWAS of tinnitus consists of 14,829 tinnitus subjects 119,600 subjects who have never experienced tinnitus [37]. One SNP (rs4906228) upstream of the RCOR1 gene showed genome-wide significant association with tinnitus $\left(P=1.7 \times 10^{8}\right)$. Together, these results suggested that GWAS can identify interesting candidate genes for tinnitus, and these candidate genes deserve further investigation.

The advantage of this study is that the selection of the case-control population is strict, with the cases and controls exposing to the same intensity and time of noise exposure in the same environmental conditions. All the subjects were male, therefore excluding the potential confounding caused by sex. However, this study also has its shortcomings. For example, the sample size in the initial GWAS discovery stage in this study is not sufficient enough to determine all possible genetic susceptibility loci associated with noise-induced tinnitus. Therefore, the potential SNPs associated with the disease may be missed in the present study. Due to the same reason, no SNP in our results reached genome-wide significance, our conclusion is therefore reasonable speculation [38].
However, our GWAS reveals enough statistical power in 65 cases and 233 controls to detect the index rs2846071 $(\mathrm{OR}=2.54$; minor allele frequency $[\mathrm{MAF}]=$ $0.341)$ and $\mathrm{rs} 10081191(\mathrm{OR}=2.33$; $\mathrm{MAF}=0.359)$, with the estimated powers to be $\sim 94 \%$ and $\sim 85 \%$, respectively (Fig. S8). Besides, the rare variation is difficult to be discovered by GWAS technology, which may also lead to the "missing heritability" of tinnitus.

\section{Conclusions}

In summary, we conducted the first GWAS of noiseinduced tinnitus in Chinese populations and identified novel loci at $11 \mathrm{q} 13.5$ and $12 \mathrm{p} 13.31$. We suggested that WNT11 and TNFRSF1A may be the susceptible genes at $11 \mathrm{q} 13.5$ and 12p13.31, respectively. Further functional studies are warranted to establish the roles of these two loci in the pathogenesis of tinnitus. These findings advanced our understanding of the genetic mechanism of noise-induced tinnitus, and might be helpful in identifying the high-risk groups of tinnitus among noise workers, and in improving the treatment of this disease.

\section{Methods \\ Study participants}

In the present study, we performed a two-stage GWAS, totally consisting of 711 male subjects of Chinese ancestry. The discovery stage contains 298 subjects who were recruited from occupational noise-exposed workers from a single factory in March, 2018 from Bengbu city in Anhui province, China (Table S1). The replication stage contains 413 subjects, who were recruited from the same factory between August, 2018 and September, 2019 (Table S1).

These workers were exposed to noise greater than 100 $\mathrm{dB}(\mathrm{dB})$ for more than $8 \mathrm{~h}$ per day for at least half a year. All the subjects underwent pure tone audiometry by qualified audiologists in a standard soundproof room using a Madsen Voyager 522 audiometer (Kastrup, Denmark) according to the standard procedures. Air conduction hearing thresholds were measured for tonal stimuli at the frequencies of $0.25,0.5,1,2,4$ and $8 \mathrm{kHz}$ $(\mathrm{kHz})$. In addition, all the subjects completed a detailed questionnaire on tinnitus, medical history and exposure to environmental risk factors, including demographic factors, noise exposure time, noise exposure intensity, a hearing threshold of both ears after noise exposure. Subjects with hearing-related complications, ear trauma, certain drugs or toxins, and otitis media were excluded.

The noise-induced tinnitus patients (cases) were defined according to the 2009 Guidelines for diagnosis and Treatment of Tinnitus (Proposal) [39]. Briefly, the subjects with persistent tinnitus after noise exposure, or with non-persistent tinnitus after noise exposure (more than 3 times) were defined as the cases. The persistent 
tinnitus was tinnitus lasting more than 6 months, which often negatively affects the patient's quality of life [39]. All the noise-induced tinnitus patients completed a detailed Tinnitus Handicap Inventory (THI) questionnaire in this study to evaluate the severity of tinnitus [40]. Subjects without tinnitus were defined as controls. According to these criteria, the discovery stage contains 65 cases and 233 controls, and the mean age of the cases (23.8) is slightly higher than that of controls (23.4) $(P=$ 0.080; Table S1). The replication stage contains 34 cases and 379 controls, and the mean age of cases (26.4) is significantly higher than that of controls $(24.5)(P=0.0070$; Table S1). All the cases and controls are males, therefore excluding the potential confounding caused by sex.

\section{Genotyping and quality controls in the discovery stage}

In the discovery stage, we genotyped all the participants using the Illumina Infinium Asian Screening Array-24 (v1.0). We performed strict quality controls for samples and SNPs to ensure the subsequent robust association tests [41]. Briefly, we removed the samples that: (i) had overall call rates of $<90 \%$; (ii) showed sex ambiguous; or (iii) were identified as outliers by the PCA. Genomewide Complex Trait Analysis software (GCTA; v1.92.2) was used for PCA to detect the outliers [42]. SNPs were retained if they had: (i) a call rate of $>90 \%$; (ii) a MAF of $>0.05$; (iii) did not map to the sex chromosomes; and (iv) $P$ values of great than $1.0 \times 10^{-4}$ in a HardyWeinberg equilibrium test. Finally, a total of 302,253 SNPs, and 65 cases and 233 controls remained for subsequent analyses (Tables S1 and S2).

\section{SNP imputation}

To increase the coverage of SNPs and get more genotypes in the discovery stage, we performed imputation based on the GWAS genotyping data in the discovery stage using the SHAPEIT (v2) [43] and IMPUTE2 (v2.3.1) [44] software. The 1000 Genomes Project data (phase 3) from all populations was used as the reference dataset. The posterior probability of 0.90 was used as the threshold of genotyping. The imputed probabilities were then converted to hard genotype calls. For the imputed SNPs, we also performed the quality controls to screen well-qualified SNPs. SNPs were retained if they had: (i) IMPUTE2 info > 0.6; (ii) a call rate of $>90 \%$; (iii) a MAF of $>0.05$; and (iv) a $P$ value of great than $1.0 \times 10^{-4}$ in a Hardy-Weinberg equilibrium test. Finally, a total of $3,830,431$ SNPs was obtained after strict quality controls among 65 cases and 233 controls in the discovery stage (Table S2). We also used the Michigan Imputation Server the includes 1654 individuals from the GenomeAsia $100 \mathrm{~K}$ Project to improve imputation since this reference panel is restricted to Asians. We, however, achieved similar results [45].
Genome-wide association analyses in the discovery stage Association analyses between each SNP and the risk of noise-induced tinnitus were performed using PLINK (v1.9) [46], which used logistic regression analyses under an additive model with adjustment for age and noise exposure time. Because we did not find any significant principal components (PCs) from the Tracy-Widom statistic, we didn't adjust for PCs in the logistic regression model. The Manhattan plot of $-\log 10$ ( $P$ values) was generated to show the associations between the SNPs and the risk of tinnitus. The quantile-quantile plot was generated to assess the potential impact of population stratification and evaluate the overall significance of the genome-wide associations. The Manhattan and quantilequantile plots were created with package qqman (version 0.1.8) in $\mathrm{R}$ (version 4.0.3). A lambda $(\lambda)$ inflation factor is given to indicate whether the systematic bias is present.

\section{SNPs selection and genotyping in the replication stage} A total of 232 SNPs with $P$ values $\leq 1.0 \times 10^{-4}$ in the discovery stage were chosen for the replication study. To select the SNPs to enter into the replication stage, we employed the tagger algorithm implemented in the Haploview (version 4.2) software to select the tag-SNPs among 232 SNPs in the study [47]. We first screened out the SNPs with $r^{2} \geq 0.8$ and MAF $\geq 0.05$ in the haplotype block with Haploview software, and then selected the SNPs with the mean maximum $r^{2}$ as the tag-SNPs in the haplotype block $[48,49]$. Thus, we achieved a total of 22 loci among these 232 SNPs. Then, these 22 tagSNPs with significant $P$ values in each locus ( $P$ values $\left.\leq 1.0 \times 10^{-4}\right)$, which were designated as the index SNPs, were selected for genotyping in the subsequent replication stage. These 22 index SNPs were genotyped using the Sequenom assays. First, locus-specific PCR and primers were designed for the 22 index SNPs using the MassARRAY Assay Design 3.0 software (Sequenom, Inc. USA). Then, approximately $15 \mathrm{ng}$ of the genomic DNA for each sample was used to genotype these SNPs. The DNA samples were amplified by multiplex PCR, and the products were then used for locus-specific single-base extension reactions. The resulting products were desalted and transferred to a 384-element SpectroCHIP array (Sequenom, Inc. USA). Allele detection was performed using MALDI-TOF-MS (Sequenom, Inc. USA). The mass spectrograms were analyzed by the MassARRAY TYPER software. The cluster patterns of the genotyping data from the Sequenom assays were visually checked to confirm their good quality. Lastly, the genotype data in the replication stage was subjected to the same quality control analyses as in the discovery stage. Among the 22 SNPs, rs148091530 was failed to be genotyped. Association analyses between each SNP and the 
risk of noise-induced tinnitus were performed using PLINK (v1.9), which used logistic regression analyses under an additive model with adjustment for age and noise exposure time. Finally, only the rs2846071 and rs4149577 were survived in the replication stage $(P<$ 0.05 and with effects in the same direction as that in the discovery stage).

\section{Genotype-expression association analyses}

Several neuroscience studies have found that the nerve changes related to tinnitus start in the cochlear nucleus and extend to the auditory cortex and other brain regions [11]; therefore, we evaluated the genotype-specific expressions for rs2846071 and rs4149577 in 13 types of human brain tissue using the eQTL analyses based on the GTEx (v8) portal [50]. The $P$ value was calculated by the "eQTL Calculator" tool on the GTEx (v8) official website. We only focused on protein-coding genes within 1 megabase $(\mathrm{Mb})$ surrounding the association signals. The $P$ value of less than 0.05 was considered to be statistically significant.

\section{Other analyses}

Details of colocalization analyses for GWAS and eQTL signals, functional annotations of the candidate SNPs and pathway enrichment analyses are provided in the Supplementary Methods.

\section{Statistical analyses}

The $\chi^{2}$ test was performed to compare the differences in clinical characteristics between the cases and controls. A fixed-effect model was used in the meta-analyses of SNPs using PLINK (v1.9) software. Cochran's Q statistic was calculated to test the between-group heterogeneity for each SNP. The potential modification effects of age on tinnitus risk were assessed by the addition of interaction terms in the logistic regression model and by separate analyses of subgroups of subjects stratified by these factors.

\section{Abbreviations}

Cl: Confidence interval; dB: decibels; eQTL: Expression quantitative trait loci; GCTA: Genome-wide Complex Trait Analysis software; GWAS: Genome-wide association study; MAF: Minor allele frequency; OR: Odds ratio; PCA: Principal component analysis; PCP: Planar cell polarity; Q-Q: Quantile-quantile; SNP: Single nucleotide polymorphism; THI: Tinnitus Handicap Inventory: TNF: Tumor necrosis factor; TNFRSF1A: Tumor necrosis factor receptor superfamily member $1 \mathrm{~A}$

\section{Supplementary Information}

The online version contains supplementary material available at https://doi. org/10.1186/s12863-021-00987-y.

Additional file 1: Supplementary Figure 1. The principal components analyses (PCA) of the population in the discovery stage in this study and reference populations from the 1,000 Genomes Project. Supplementary Figure $\mathbf{2}$. The genotypes of rs 2846071 are significantly associated with the expression levels of WNT11 in several types of brain tissues from GTEx. Supplementary Figure 3. Colocalization analyses of the association signals from GWAS and brain eQTL data at the 11q13.5 and 12p13.31 loci. Supplementary Figure 4. Chromatin state segmentations for rs 1800692 and rs4149570 using the ENCODE data. Supplementary Figure 5. The genotypes of rs4149577 are significantly associated with the expression levels of TNFRSFIA in several types of brain tissue from GTEx. Supplementary Figure 6. Proxy plots for 11q13.5 and 12p13.31 regions in Chinese Han Chinese and European populations.

Supplementary Figure 7. Linkage disequilibrium plots for 11q13.5 and 12 p13.31 regions in Chinese Han Chinese and European populations. Supplementary Figure 8. Power to detect the genetic effects of rs2846071 and rs4149577. Supplementary Table 1. Summary of the case/control populations used in this study. Supplementary Table 2. Summary of the genotyped and imputed SNPs in the discovery stage. Supplementary Table 3. Summary of the SNPs that have been reported to be associated with tinnitus in previous studies.

Supplementary Table 4. Summary of the top 22 SNPs in the discovery stage. Supplementary Table 5. Primers used for SNPs genotyping in the replication stage. Supplementary Table 6. Summary of the association results in the replication stage. Supplementary Table $\mathbf{7 .}$ Stratification analyses of rs 2846071 and rs4149577 by age.

Supplementary Table 8. The predicted functional relevance of rs2846071, rs4149577 and the other SNPs in strong or moderate LD with them. Supplementary Table 9. Pathway analyses based on iGSEA4GWAS. Supplementary Table 10. The allele and genotype frequencies of rs 2846071 and rs4149577 in different populations.

\section{Acknowledgments}

The authors thank all the patients participating in this study.

\section{Fundings}

This work was supported by grants from the National Natural Science Foundation of China (No. 91440206, 31771397 and 81702370), Special Foundation from the China Postdoctoral Science Foundation (No. 2018 T111147), Beijing Institute of Radiation Medicine (BIRM) Innovation Fund (BIOX0105) and General Financial Grant from the China Postdoctoral Science Foundation (2017 M613414). The funding bodies played no role in the design of the study and collection, analysis, and interpretation of data and in writing the manuscript.

\section{Authors' contributions}

$G Z$ and $Y L$ were the principal investigators who conceived the study and obtained financial supports. GZ, YN and YL designed the study. CX analyzed the data. YN conducted sample selection and data management, JP, CY and YW performed the statistical analyses, GZ, YL and CX. drafted the manuscript. GZ approved the final version of the manuscript. All authors have read and approved the manuscript.

\section{Availability of data and materials}

The datasets generated during and/or analyzed during the current study are available in the repository, http://cbportal.org/pubfiles/lyf_tinnus_298.logistic.

\section{Declarations}

Ethics approval and consent to participate

This study was performed with the approval of the Medical Ethical Committee of Beijing Institute of Radiation Medicine (Beijing, China) and the General Hospital of PLA (Beijing, China). Written informed consent was obtained from each participant. The investigators were blind to the case/ control status of subjects during all genotyping experiments. All methods were carried out in accordance with relevant guidelines and regulations.

Consent for publication

Not Applicable.

Competing interests

The authors declared no competing interests. 


\section{Author details}

Medical College of Guizhou University Guiyang City 550025 China ${ }^{2}$ Department of Ambulatory Medicine, The First Medical Center of PLA General Hospital, Beijing 100853, China. ${ }^{3}$ State Key Laboratory of Proteomics, National Center for Protein Sciences, Beijing Institute of Radiation Medicine, Beijing 100850, China. ${ }^{4}$ Collaborative Innovation Center for Personalized Cancer Medicine, Center for Global Health, School of Public Health, Nanjing Medical University, Nanjing City 210029, China.

Received: 22 April 2021 Accepted: 25 August 2021

Published online: 06 September 2021

\section{References}

1. Lopez-Escamez JA, Bibas T, Cima RF, Van de Heyning P, Knipper M, Mazurek $B$, et al. Genetics of tinnitus: An emerging area for molecular diagnosis and drug development. Front Neurosci. 2016;10:377.

2. Bing D, Lee SC, Campanelli D, Xiong H, Matsumoto M, Panford-Walsh R, et al. Cochlear NMDA receptors as a therapeutic target of noise-induced tinnitus. Cell Physiol Biochem. 2015;35(5):1905-23.

3. Baguley D, McFerran D, Hall D. Tinnitus. Lancet. 2013;382(9904):1600-7.

4. Shargorodsky J, Curhan GC, Farwell WR. Prevalence and characteristics of tinnitus among US adults. Am J Med. 2010;123(8):711-8.

5. Yankaskas K. Prelude: noise-induced tinnitus and hearing loss in the military. Hear Res. 2013;295:3-8.

6. Kim HJ, Lee HJ, An SY, Sim S, Park B, Kim SW, et al. Analysis of the prevalence and associated risk factors of tinnitus in adults. PLoS One. 2015: 10(5):e0127578.

7. Stouffer JL, Tyler RS. Characterization of tinnitus by tinnitus patients. J Speech Hear Disord. 1990;55(3):439-53.

8. Bogo R, Farah A, Karlsson KK, Pedersen NL, Svartengren M, Skjonsberg A. Prevalence, incidence proportion, and heritability for tinnitus: a longitudinal twin study. Ear Hear. 2017;38(3):292-300.

9. Vona B, Nanda I, Shehata-Dieler W, Haaf T. Genetics of tinnitus: still in its infancy. Front Neurosci. 2017;11:236.

10. Gilles A, Van Camp G, Van de Heyning P, Fransen E. A pilot genome-wide association study identifies potential metabolic pathways involved in tinnitus. Front Neurosci. 2017;11:71.

11. Shore SE, Roberts LE, Langguth B. Maladaptive plasticity in tinnitus--triggers, mechanisms and treatment. Nat Rev Neurol. 2016;12(3):150-60.

12. Kalappa BI, Brozoski TJ, Turner JG, Caspary DM. Single unit hyperactivity and bursting in the auditory thalamus of awake rats directly correlates with behavioural evidence of tinnitus. J Physiol. 2014;592(22):5065-78.

13. Gong Y, Greenbaum J, Deng HW. A statistical approach to fine-mapping for the identification of potential causal variants related to human intelligence. J Hum Genet. 2019;64(8):781-7.

14. Hu Y, Sun JY, Zhang Y, Zhang H, Gao S, Wang T, et al. Liu G: rs1990622 variant associates with Alzheimer's disease and regulates TMEM106B expression in human brain tissues. BMC Med. 2021;19(1):11.

15. Zhang K, Chang S, Guo L, Wang J. I-GSEA4GWAS v2: a web server for functional analysis of SNPS in trait-associated pathways identified from genome-wide association study. Protein Cell. 2015;6(3):221-4.

16. Ruel J, Chabbert C, Nouvian R, Bendris R, Eybalin M, Leger CL, et al. Salicylate enables cochlear arachidonic-acid-sensitive NMDA receptor responses. J Neurosci. 2008;28(29):7313-23.

17. Gossman DG, Zhao HB. Hemichannel-mediated inositol 1,4,5-trisphosphate (IP3) release in the cochlea: a novel mechanism of IP3 intercellular signaling Cell Commun Adhes. 2008;15(4):305-15.

18. Jayasena CS, Ohyama T, Segil N, Groves AK. Notch signaling augments the canonical Wnt pathway to specify the size of the otic placode. Development. 2008;135(13):2251-61.

19. Romero-Carvajal A, Navajas Acedo J, Jiang L, Kozlovskaja-Gumbriene A, Alexander R, Li H, et al. Regeneration of sensory hair cells requires localized interactions between the notch and Wnt pathways. Dev Cell. 2015;34(3): 267-82.

20. Wu J, Li W, Lin C, Chen Y, Cheng C, Sun S, et al. Co-regulation of the notch and Wnt signaling pathways promotes supporting cell proliferation and hair cell regeneration in mouse utricles. Sci Rep. 2016;6:29418.

21. Dinh CT, Bas E, Chan SS, Dinh JN, Vu L, Van De Water TR. Dexamethasone treatment of tumor necrosis factor-alpha challenged organ of Corti explants activates nuclear factor kappa B signaling that induces changes in gene expression that favor hair cell survival. Neuroscience. 2011;188:157-67.
22. Freedman ML, Monteiro AN, Gayther SA, Coetzee GA, Risch A, Plass C, et al. Principles for the post-GWAS functional characterization of cancer risk loci. Nat Genet. 2011;43(6):513-8.

23. Dimas AS, Deutsch S, Stranger BE, Montgomery SB, Borel C, Attar-Cohen H, et al. Common regulatory variation impacts gene expression in a cell typedependent manner. Science. 2009;325(5945):1246-50.

24. Arisan ED, Rencuzogullari O, Freitas IL, Radzali S, Keskin B, Kothari A, et al. Upregulated Wnt-11 and miR-21 expression trigger Epithelial Mesenchymal transition in aggressive Prostate Cancer Cells. Biology. 2020;9(3):52.

25. Dutta S, Sriskanda S, Boobalan E, Alur RP, Elkahloun A. Brooks BP: nlz1 is required for cilia formation in zebrafish embryogenesis. Dev Biol. 2015; 406(2):203-11.

26. de Almeida-Silva I, de Oliveira JA, Rossato M, Salata FF, Hyppolito MA. Spontaneous reversibility of damage to outer hair cells after sodium salicylate induced ototoxicity. J Laryngol Otol. 2011;125(8):786-94.

27. Rios AC, Marcelle C, Serralbo O. Gene loss-of-function and live imaging in chick embryos. Methods Mol Biol. 2012;839:105-17.

28. Shi F, Hu L, Jacques BE, Mulvaney JF, Dabdoub A. Edge AS: beta-catenin is required for hair-cell differentiation in the cochlea. J Neurosci. 2014;34(19): 6470-9.

29. Liu L, Chen Y, Qi J, Zhang Y, He Y, Ni W, et al. Wnt activation protects against neomycin-induced hair cell damage in the mouse cochlea. Cell Death Dis. 2016;7:e2136.

30. Navajas Acedo J, Voas MG, Alexander R, Woolley T, Unruh JR, Li H, et al. PCP and Wnt pathway components act in parallel during zebrafish mechanosensory hair cell orientation. Nat Commun. 2019;10(1):3993.

31. Hapil FZ, Copuroglu FE, Ertosun MG, Mert U, Ozes D, Ozes ON. Negative regulation of TNFR1 signaling via PKA-mediated phosphorylation of TNFR1. J Interfer Cytokine Res. 2020;40:225-35.

32. Bas E, Van De Water TR, Gupta C, Dinh J, Vu L, Martinez-Soriano F, et al. Efficacy of three drugs for protecting against gentamicin-induced hair cell and hearing losses. Br J Pharmacol. 2012;166(6):1888-904.

33. Ren Y, Stankovic KM. The role of tumor necrosis factor alpha (TNFalpha) in hearing loss and vestibular Schwannomas. Curr Otorhinolaryngol Rep. 2018 6(1):15-23.

34. Wang W, Zhang LS, Zinsmaier AK, Patterson G, Leptich EJ, Shoemaker SL, et al. Neuroinflammation mediates noise-induced synaptic imbalance and tinnitus in rodent models. PLoS Biol. 2019;17(6):e3000307.

35. Felder CC, Kanterman RY, Ma AL, Axelrod J. Serotonin stimulates phospholipase A2 and the release of arachidonic acid in hippocampal neurons by a type 2 serotonin receptor that is independent of inositolphospholipid hydrolysis. Proc Natl Acad Sci U S A. 1990;87(6):218791.

36. Crummer RW, Hassan GA. Diagnostic approach to tinnitus. Am Fam Physician. 2004;69(1):120-6.

37. Wells HRR, Abidin FNZ, Freidin MB, Williams FMK, Dawson SJ. Genome-wide association study suggests that variation at the RCOR1 locus is associated with tinnitus in UK biobank. Sci Rep. 2021;11(1):6470

38. Asif H, Alliey-Rodriguez N, Keedy S, Tamminga CA, Sweeney JA, Pearlson G, et al. GWAS significance thresholds for deep phenotyping studies can depend upon minor allele frequencies and sample size. Mol Psychiatry. 2020.

39. Tunkel DE, Bauer CA, Sun GH, Rosenfeld RM, Chandrasekhar SS, Cunningham ER Jr, et al. Clinical practice guideline: tinnitus. Otolaryngol Head Neck Surg. 2014;151(2 Suppl):S1-S40.

40. Job A, Cardinal F, Michel H, Klein C, Ressiot E, Gauthier J. Tinnitus and associated handicaps in the French Mountain artillery: assessment by the tinnitus handicap inventory. Mil Med. 2018;183(9-10):e302-6.

41. Li Y, Zhai $Y$, Song $Q$, Zhang $H$, Cao P, Ping J, et al. Genome-wide association study identifies a new locus at 7q21.13 associated with hepatitis B virusrelated hepatocellular carcinoma. Clin Cancer Res. 2018;24(4):906-15.

42. Yang J, Lee SH, Goddard ME, Visscher PM. GCTA: a tool for genome-wide complex trait analysis. Am J Hum Genet. 2011;88(1):76-82.

43. Delaneau O, Coulonges C, Zagury JF. Shape-IT: new rapid and accurate algorithm for haplotype inference. BMC Bioinformatics. 2008;9:540.

44. van Leeuwen EM, Kanterakis A, Deelen P, Kattenberg MV. Genome of the Netherlands C, Slagboom PE, de Bakker PI, Wijmenga C, Swertz MA, Boomsma DI et al: population-specific genotype imputations using minimac or IMPUTE2. Nat Protoc. 2015;10(9):1285-96.

45. GenomeAsia KC. The GenomeAsia 100K project enables genetic discoveries across Asia. Nature. 2019;576(7785):106-11. 
46. Renteria ME, Cortes A, Medland SE. Using PLINK for genome-wide association studies (GWAS) and data analysis. Methods Mol Biol. 2013;1019: 193-213.

47. Barrett JC, Fry B, Maller J, Daly MJ. Haploview: analysis and visualization of LD and haplotype maps. Bioinformatics. 2005;21(2):263-5.

48. Carlson CS, Eberle MA, Rieder MJ, Yi Q, Kruglyak L, Nickerson DA. Selecting a maximally informative set of single-nucleotide polymorphisms for association analyses using linkage disequilibrium. Am J Hum Genet. 2004; 74(1):106-20.

49. de Bakker PI, Yelensky R, Pe'er I, Gabriel SB, Daly MJ, Altshuler D. Efficiency and power in genetic association studies. Nat Genet. 2005;37(11):1217-23.

50. Consortium GT. Human genomics. The genotype-tissue expression (GTEx) pilot analysis: multitissue gene regulation in humans. Science. 2015. 348(6235):648-60.

\section{Publisher's Note}

Springer Nature remains neutral with regard to jurisdictional claims in published maps and institutional affiliations.

Ready to submit your research? Choose BMC and benefit from:

- fast, convenient online submission

- thorough peer review by experienced researchers in your field

- rapid publication on acceptance

- support for research data, including large and complex data types

- gold Open Access which fosters wider collaboration and increased citations

- maximum visibility for your research: over $100 \mathrm{M}$ website views per year

At BMC, research is always in progress.

Learn more biomedcentral.com/submissions 